\title{
A citizens workgroup helps researchers reflect on their work
}

\author{
Roman Seidl $^{1}$, Cord Drögemüller ${ }^{1}$, Pius Krütlii $^{2}$, and Clemens Walther ${ }^{1}$ \\ ${ }^{1}$ Institute of Radioecology and Radiation Protection, Leibniz University Hanover, 30419 Hanover, Germany \\ ${ }^{2}$ USYS TdLab, ETH Zurich, 8092 Zurich, Switzerland \\ Correspondence: Cord Drögemüller (droegemueller@irs.uni-hannover.de)
}

Published: 10 November 2021

\begin{abstract}
In our transdisciplinary project (http://www.transens.de) several academic disciplines work on questions and solutions for high-level nuclear waste disposal in Germany. Adding to this interdisciplinary setting, in our sub-project on trust, we have enlisted a group of 16 citizens (citizens workgroup, CWG), reflecting with us on our research with regard to research questions and approaches. In this talk, we present results from a joint workshop of researchers and the CWG on the role of trust in scientists. We want to understand how participants perceive and relate to science and how this may affect trust in scientists and experts. During a first workshop (conducted online in March 2021), the CWG members were allocated to three breakout groups (1)-(3) to discuss three guiding questions:
\end{abstract}

a. What expectations do you have of a scientist from Social Sciences and Humanities (SSH)/Natural Sciences and Engineering (NSE) in the TRANSENS project?

b. What characterizes a scientist from SSH/NSE that you trust?

c. What would have to happen for you to no longer trust him/her?

The group in breakout room (1) was asked to answer questions (a)-(c) for a fictitious SSH researcher. The group in breakout room (2) was asked to do the same for a fictitious NSE researcher. The group in breakout room (3) was asked whether they perceived differences in the trust of SSH and NSE researchers.

For SSH we found that a scientist should be sympathetic and non-condescending, represent a neutral point of view, and consider all opinions. Remarkably, discussants in this group struggled to define a role for SSH scientists in high-level waste disposal research. Some participants ascribed SSH a moderating or mediating role. If necessary (e.g. communication of results), mediation between the NSE and the public can be added. SSH scientists may train other scientists with regard to their performance.

Participants stated that scientists from NSE should present information in full and clarify the current state of research (provisional nature of knowledge acknowledged). A scientist should not conceal any information, uncertainties, or risks, be neutral and objective, have experience in the field (professionalism, experience, reputation), and not exclusively reproduce one's own opinion or spread untruths. In all groups, participants judged the NSE as "harder", more serious and more tangible. It was also discussed that the scientist's appearance is of great importance and that a scientist can "pick up" the audience in an exciting way when communicating. Personal experience was mentioned several times in all groups as a basis for trust. These results match findings on the effect of value similarity (Siegrist et al., 2000, and own survey in 2020 in Germany): If a person is perceived as advocating similar values, it is more likely that I trust that person. Personal experience of - among other issues - similar values may increase trust.

Moreover, and importantly, trust also emerges when participants know exactly how issues will be considered, e.g. if input from the CWG is considered or not - and if not: why? In general, participants want to be taken seriously. Thus, transparent and binding rules for all participants may be key for a relationship of trust. 
Kurzfassung. In unserem transdisziplinären Projekt (http://www.transens.de) arbeiten mehrere wissenschaftliche Disziplinen an Fragestellungen und Lösungsansätzen für die Entsorgung hochradioaktiver Abfälle in Deutschland. Ergänzend zu diesem interdisziplinären Setting haben wir für unser Teilprojekt TRUST eine Gruppe von 16 Bürgern (Arbeitsgruppe Bevölkerung, AGBe) gewonnen, die unsere Arbeit hinsichtlich Forschungsfragen und -ansätzen mit uns reflektieren. In diesem Vortrag präsentieren wir Ergebnisse gemeinsamer Workshops von Forschern und der AGBe zur Rolle von Vertrauen in Wissenschaftler. Wir wollen verstehen, wie die Teilnehmer Wissenschaft wahrnehmen, wie sie ihr gegenüber eingestellt sind und wie sich dies auf das Vertrauen in Wissenschaftler und Experten auswirken kann. In einem ersten Workshop (im März 2021 online durchgeführt) wurden die AGBe-Mitglieder in drei Arbeitsgruppen (1-3) eingeteilt, um drei Leitfragen zu diskutieren:

a. Welche Erwartungen haben Sie an einen Geistes- oder Sozialwissenschaftler (SoWi)/Naturwissenschaftler oder Ingenieur (NaWi) im Projekt TRANSENS?

b. Was zeichnet eine Sozial-/Geisteswissenschaftlerin/eine Naturwissenschaftlerin oder Ingenieurin aus, der Sie vertrauen?

c. Was müsste passieren, dass Sie ihm/ihr nicht mehr vertrauen?

Arbeitsgruppe 1 wurde gebeten, die Fragen (a)-(c) für einen fiktiven SoWi-Wissenschaftler zu beantworten, Arbeitsgruppe 2 sollte dasselbe für einen fiktiven NaWi-Wissenschaftler umsetzen. Arbeitsgruppe 3 wurde gefragt, ob die Teilnehmer Unterschiede im Vertrauen gegenüber SoWi- bzw. NaWi-Wissenschaftler wahrnehmen.

Für SoWi stellten wir fest, dass ein solcher Wissenschaftler sympathisch und nicht herablassend sein, einen neutralen Standpunkt vertreten und alle Meinungen berücksichtigen sollte. Bemerkenswerterweise hatten die Diskussionsteilnehmer in dieser Gruppe Schwierigkeiten, eine Rolle für SoWi-Wissenschaftler in der Forschung zur Entsorgung hochradioaktiver Abfälle zu definieren. Einige Teilnehmer schrieben SoWi-Wissenschaftlern eine moderierende oder vermittelnde Rolle zu. Bei Bedarf (z. B. Ergebniskommunikation) kann eine Vermittlung zwischen NaWi und der Öffentlichkeit hinzukommen. Die SoWi-Wissenschaftler können andere Wissenschaftler hinsichtlich ihrer Kommunikationsskills ausbilden.

Die Teilnehmer gaben an, dass NaWi-Wissenschaftler Informationen vollständig präsentieren und den aktuellen Forschungsstand verdeutlichen sollten (inklusive einem vorläufigen Charakter der Erkenntnisse). Ein Wissenschaftler sollte keine Informationen, Unsicherheiten oder Risiken verschweigen, neutral und objektiv sein, Erfahrung auf dem betreffenden Gebiet haben (Professionalität, Erfahrung, Reputation) und nicht ausschließlich die eigene Meinung wiedergeben oder Unwahrheiten verbreiten. In allen Gruppen beurteilten die Teilnehmer die NaWi als „härter“, ernsthafter und greifbarer. Diskutiert wurde auch, dass das Erscheinungsbild des Wissenschaftlers von großer Bedeutung ist und dieser das Publikum bei der Kommunikation spannend ,abholen" kann. Persönliche Erfahrungen wurden in allen Gruppen mehrfach als Vertrauensbasis genannt. Diese Ergebnisse stimmen mit Befunden zum Effekt der Werteähnlichkeit überein (Siegrist et al., 2000, und eigene Studie 2020 in Deutschland): Wenn eine Person als Befürworter ähnlicher Werte wahrgenommen wird, ist es wahrscheinlicher, dass ich ihr vertraue. Persönliche Erfahrungen - u. a. - von ähnlichen Werten können das Vertrauen stärken.

Darüber hinaus, und das ist wichtig, entsteht Vertrauen auch dann, wenn die Teilnehmer genau wissen, wie Themen behandelt werden, z. B. ob Input aus der AGBe berücksichtigt wird oder nicht - und wenn nein: warum? Generell wollen die Teilnehmer ernst genommen werden. Somit können transparente und verbindliche Regeln für alle Beteiligten der Schlüssel für ein Vertrauensverhältnis sein. 
Financial support. This research has been supported by the Bundesministerium für Wirtschaft und Technologie (grant no. 02E11849F), the Volkswagen Foundation, and the Niedersächsische Ministerium für Wissenschaft und Kultur.

\section{References}

Siegrist, M., Cvetkovich, G., and Roth, C.: Salient value similarity, social trust, and risk/benefit perception, Risk Anal., 20, 353-362, https://doi.org/10.1111/0272-4332.203034, 2000. 